\title{
Genome Wide Host Gene Expression Analysis in Chicken Lungs Infected with Avian Influenza Viruses
}

\author{
Pradip B. Ranaware ${ }^{1 \oplus}$, Anamika Mishra ${ }^{1 \oplus}$, Periyasamy Vijayakumar ${ }^{1 \oplus}$, Pradeep \\ N. Gandhale ${ }^{1}$, Himanshu Kumar ${ }^{2}$, Diwakar D. Kulkarni ${ }^{1}$, Ashwin Ashok Raut ${ }^{1 *}$ \\ 1 Pathogenomics Laboratory, ICAR -National Institute of High Security Animal Diseases, OIE Reference lab \\ for Avian Influenza, Bhopal, Madhya Pradesh, India, 2 Laboratory of Immunology, Department of Biological \\ Sciences, Indian Institute of Science Education and Research, Bhopal, Madhya Pradesh, India \\ ه These authors contributed equally to this work. \\ * ashwin.hsadl@gmail.com
}

\section{Gopenaccess}

Citation: Ranaware PB, Mishra A, Vijayakumar P, Gandhale PN, Kumar H, Kulkarni DD, et al. (2016) Genome Wide Host Gene Expression Analysis in Chicken Lungs Infected with Avian Influenza Viruses. PLOS ONE 11(4): e0153671. doi:10.1371/journal. pone. 0153671

Editor: Niyaz Ahmed, University of Hyderabad, INDIA

Received: December 2, 2015

Accepted: April 1, 2016

Published: April 12, 2016

Copyright: @ 2016 Ranaware et al. This is an open access article distributed under the terms of the Creative Commons Attribution License, which permits unrestricted use, distribution, and reproduction in any medium, provided the original author and source are credited.

Data Availability Statement: The microarray data have been deposited in NCBI Gene Expression Omnibus (Accession number GSE65231).

Funding: This work was funded by the Department of Biotechnology (grant number: BT/IN/Indo-UK/ FADH/48/AM/2013) and ICAR-National Institute of High Security Animal Disease (Institute funded research project). The funders had no role in study design, data collection and analysis, decision to publish, or preparation of the manuscript.

\section{Abstract}

The molecular pathogenesis of avian influenza infection varies greatly with individual bird species and virus strain. The molecular pathogenesis of the highly pathogenic avian influenza virus (HPAIV) or the low pathogenic avian influenza virus (LPAIV) infection in avian species remains poorly understood. Thus, global immune response of chickens infected with HPAI H5N1 (A/duck/India/02CA10/2011) and LPAI H9N2 (A/duck/India/249800/2010) viruses was studied using microarray to identify crucial host genetic components responsive to these infection. HPAI H5N1 virus induced excessive expression of type I IFNs (IFNA and IFNG), cytokines (IL1B, IL18, IL22, IL13, and IL12B), chemokines (CCL4, CCL19, CCL10, and CX3CL1) and IFN stimulated genes (OASL, MX1, RSAD2, IFITM5, IFIT5, GBP 1 , and EIF2AK) in lung tissues. This dysregulation of host innate immune genes may be the critical determinant of the severity and the outcome of the influenza infection in chickens. In contrast, the expression levels of most of these genes was not induced in the lungs of LPAI H9N2 virus infected chickens. This study indicated the relationship between host immune genes and their roles in pathogenesis of HPAIV infection in chickens.

\section{Introduction}

Avian influenza virus (AIV) is classified based on their virulence in chickens into low pathogenic avian influenza virus (LPAIV) and highly pathogenic avian influenza virus (HPAIV) [1]. Pathogenicity differences observed between HPAIV and LPAIV might be related to specific characteristics of virus strains, tissue tropism and host responses [2]. Low pathogenic avian influenza virus usually produces subclinical infections in chickens [3]. In contrast, highly pathogenic avian influenza infections lead to rapid onset of severe, contagious systemic disease with $100 \%$ mortality within 48 hours of infection [3].

LPAIV mainly replicate in the respiratory and the digestive tract of chicken. However, Post et al reported the presence of H7N1 LPAIV in organs beyond the respiratory and the gastrointestinal tract indicating that the virus could spread systemically after an intranasal/intratracheal 
Competing Interests: The authors have declared that no competing interests exist. infection [2]. HPAIV have a polybasic hemagglutinin (HA) cleavage site sensitive to ubiquitous proteases which enables them to replicate in multiple organs, thereby causing severe systemic disease with high mortality in gallinaceous poultry $[3,4]$. Although cleavage activation of HA is necessary for the pathogenicity of HPAIV, host factors involved in the molecular pathogenesis of HPAIV infection in chickens have not yet been identified [5].

H9N2 virus is among the most commonly seen influenza viruses in domestic poultry populations [6] and it is unique among LPAIV in that they infect a wide variety of species including various domestic poultry species, pigs, and humans [7, 8]. Because H9N2 viruses infect a number of species, crossing the species barriers, it holds potential to impact as emerging virus $[8,9]$. Hence, it warrants further studies on the molecular pathogenesis of H9N2 viruses in both humans and avian species [6].

Dysregulation of the innate immune response may be a critical determinant of the severity and the outcome of the influenza infection $[10,11]$. Dysregulation of cytokines and chemokines in lungs is reported to cause tissue damage and high mortality in mice [12,13], ferrets [14], macaques [15, 16] and in in vitro infection models [17]. Little is known about dysregulation of cytokines and chemokines in chickens after HPAIV or LPAIV infection [18, 19]. Previous studies do not support the hypothesis that dysregulation of proinflammatory cytokines and chemokines are the critical determinant for the severity and the outcome of the influenza infection in chickens infected with HPAIVs $[18,20]$. Only few studies have compared the immune response in chickens to LPAIV and HPAIV infection $[2,5,18,20]$ and their focus was on a very limited number of genes using RT qPCR. As a result, these studies do not give a global overview of interactions between the host (chickens) and the different pathotypes of influenza virus. Most of the current host-pathogen interaction research is inclined towards human model systems [21] and the knowledge of the host-pathogen interaction and the molecular mechanisms underlying the pathogenesis of HPAIV or LPAIV infection in avian species is still merger. Hence, there is a need to understand the virus-host interactions in avian species, because the wild and domestic birds are act as the reservoirs for most of the influenza A viruses [22].

In this study, we show that HPAI H5N1 virus infection in chickens intensely triggers the genes related to inflammatory and innate immune responses. However, this intense host antiviral response is unsuccessful in controlling the rapidly progressing infection and results in high mortality in chickens. In contrast, the expression levels of most of these genes remain unchanged in the lung tissue of chickens infected with LPAI H9N2 virus. Our microarray dataset provides insight into the potential molecular pathogenesis and also reveals the molecular basis of differential response of chicken to different pathotypes of AIV.

\section{Materials and Methods}

\section{Ethics statement}

The animal experiments were carried out at Biosafety level 3+ containment facility of ICARNational Institute of High Security Animal Diseases (ICAR-NIHSAD), Bhopal, India. The experiments were approved by the institutional animal ethics committee of ICAR-NIHSAD (Approval no. 68/IAEC/HSADL/12 dated 11.05.2012), and performed under the guidance of the Committee for the Purpose of Control and Supervision of Experiments on Animals (CPCSEA), Ministry of Environment and Forests, Govt. of India.

\section{Viruses}

Avian influenza LPAI H9N2 virus (A/duck/India/249800/2010) and HPAI H5N1 virus (A/duck/India/02CA10/2011) were obtained from avian Influenza virus repository of 
ICAR-NIHSAD, Bhopal, India. The stock virus was prepared by propagating and titrating the viruses in the allantoic cavities of 12-day-old embryonated duck eggs.

\section{Animal experimental design}

A total of nine, six weeks old, specific pathogen-free chicken were divided into three groups of three birds each. Chicken of group 1 were intranasally inoculated with $10^{6} \mathrm{EID}_{50}$ of the HPAI $\mathrm{H} 5 \mathrm{~N} 1$ virus (A/duck/India/02CA10/2011). Group 2 was inoculated with $10^{6} \mathrm{EID}_{50}$ of the LPAI H9N2 virus (A/duck/India/249800/2010). Chicken in control group were inoculated with phosphate buffer saline (PBS). The birds were observed daily for clinical signs. The clinical symptoms like dullness, lacrimation, cyanotic combs and wattles gradually appeared and were full blown by 2 days post infection (dpi) in HPAI H5N1 infected chicken. Mild clinical symptoms like depression, decreased feed and water consumption and ruffled feathers indicating sickness were observed in LPAI H9N2 virus infected chicken at 6 dpi. The birds from HPAI $\mathrm{H} 5 \mathrm{~N} 1$ virus infected group were sacrificed at 2 (dpi) and the birds from LPAI H9N2 virus infected group and control group were sacrificed at $6 \mathrm{dpi}$. None of the birds inoculated with either virus died before the end points of experiments. All birds were sacrificed by cervical dislocation. Lung tissues were collected in RNA later from all sacrificed birds and stored at $-80^{\circ} \mathrm{C}$. The virus infection in lungs was confirmed by tissue inoculation in embryonated chicken eggs and hemagglutination (HA) assay.

\section{Total RNA isolation and microarray hybridization}

Total RNA was isolated from 2 lung tissue samples from control group and 3 lung tissue samples each from group 1 and group 2 using TRIzol reagent (Invitrogen, USA) with the Qiagen's RNeasy minikit (Qiagen, Germany). The integrity of RNA was checked using the Bioanalyzer. The Custom Chicken 8x60k array on Agilent platform (AMADID G4102A_059389) was synthesized from Genotypic Technology Pvt. Ltd by printing the sets of oligonucleotide. Apart from the 43603 probes present in the commercially available chicken oligio Micryoarray AMADIA: $026441(4 \mathrm{x} 44 \mathrm{~K})$, this custom array $(8 \times 60 \mathrm{k})$ had 14256 additional probes. These additional probes included ESTs of brain, liver, and lung tissues and the genes listed in Influenza A pathway in Kyoto Encyclopedia of Genes and Genomes (KEGG). The customization was done to enhance the depth of expression profiling. Total RNA was labeled using Agilent Quick Amp labeling kit by standard procedure. cRNA was purified using Qiagen RNeasy column. Concentration and amount of dye incorporated into labeled cRNA was determined using Nanodrop ND-1000 UV-VIS spectrophotometer. Samples that passed the QC for specific activity were taken for hybridization. $600 \mathrm{ng}$ of labeled cRNA were hybridized on the specific arrays using the Gene Expression Hybridization kit in Sure Hybridization champers at $65^{\circ} \mathrm{C}$ for $16 \mathrm{hrs}$. Hybridized slides were washed using Agilent Gene Expression wash buffers. Washed microarray slides were scanned on a GS600D scanner (Agilent Technologies).

\section{Microarray data analysis}

Data extraction from images was done using Feature Extraction software version 10.7. Percentile shift normalization method was used for normalization, where the locations of all the spot intensities in an array were adjusted. The normalized raw data was then statistical analyzed using GeneSpring GX 12.5 software (Agilent) to identify differentially expressed genes. The microarray data have been deposited in NCBI Gene Expression Omnibus (Accession number GSE65231). The normalized raw data results obtained with HPAI- or LPAI-infected lungs were compared to those obtained with control. We compared both the pathotypes with the control, in order to study the molecular basis of response to infection with these individual 
virus pathotypes. Gene expression ratios compared with the reference control were calculated and $\log 2$ transformed. All the fold change expression values represented in the manuscript are provided as $\log$ base 2 . The $\mathrm{p}$-value $\leq 0.05$ and fold change $\geq 1$ ( $\log 2$ transformed value) was used to determine significant differential expression. Functional classification of the genes was performed for gene ontology (GO) in Database for Annotation, Visualization and Integrated Discovery (DAVID) and pathway analysis in Kyoto Encyclopedia of Genes and Genomes (KEGG). The GO terms of immune related biological process and molecular function with $\mathrm{p}$ value $<0.05$ are discussed. The KEGG pathways involved in immune related function and viral pathogenesis were highlighted for understanding the host response to HPAI H5N1 and LPAI H9N2 infection in chicken.

\section{RT qPCR Assays}

The differential expression data was validated by one step RT qPCR. Total RNA was isolate using TRIzol @ Reagent (Invitrogen, USA) with the Qiagen's RNeasy minikit (Qiagen, Germany) from the same lung tissue samples used for the microarray analysis. Primers used for RT qPCR were as follows: $\beta$-actin, IFNA, IFNG, IFNAR2, STAT3, IRF1, and IRF10 [23], ILIB [6], TLR3, and TLR-15 [24], and Mx1 [25]. RT qPCR was done on StepOnePlus Real-Time PCR System (Applied Biosystems) using SYBR Green chemistry. The following amplification conditions were used: $2 \mathrm{~min}$ at $50^{\circ} \mathrm{C}, 10 \mathrm{~min}$ at $95^{\circ} \mathrm{C}$, followed by 40 amplification cycles ( 15 sec at $95^{\circ} \mathrm{C}$ and $1 \mathrm{~min}$ at $60^{\circ} \mathrm{C}$ ). The data obtained from the RT-qPCR was analysed by Schmittegen and Livak method [26]. The data was normalized using $\beta$-actin as the internal control gene. The $\Delta \Delta \mathrm{Ct}$ value was calculated by difference in normalized Ct value $(\Delta \mathrm{Ct})$ from infected samples to the $\Delta \mathrm{Ct}$ from non-infected samples. The $\Delta \Delta \mathrm{Ct}$ value is transformed into $2^{-\Delta \Delta \mathrm{Ct}}$ value as the estimated gene expression fold change value.

\section{Results}

\section{Host gene response to HPAI H5N1 virus infection}

Agilent chicken microarray chips were used to identify genes contributing to the innate immune response to HPAI H5N1 influenza virus infected lung tissues. Hemagglutinin titer of the HPAI H5N1 virus (A/duck/India/02CA10/2011) in infected lung tissues was $2^{8}$. As compared to the control, approximately 5550 genes were significantly differentially expressed at the cut-off of $\mathrm{p}$-value $\leq 0.05$ and fold change $\geq 1$ ( $\log 2$ transformed value) in HPAIV infected lungs tissues. Of these, 4785 genes were upregulated and 765 genes were down regulated (Table 1). We identified several immune genes upregulated including TLR3, TLR6, TLR15, IFIH1/MDA, NLRC5, MX1, IFITM5, OAS, TRIM25, SOCS3, STAT1, IL1B, IL18, IL13, IFNA, IFNG etc. and downregulated including IL12RB2, TRIM13, TGFB3, ADAT2 etc. in the lung tissue of chickens infected with HPAI H5N1 virus. The results of the microarray data were supported using RT qPCR (Fig 1). The gene expression fold-change values in microarray data were not identical to RT qPCR data; however the overall trend in gene expression changes was consistent in both dataset. Similar kinds of discrepancy in gene expression fold change values

Table 1. Differentially expressed genes in response to infection with HPAIV H5N1 and LPAIV H9N2 virus.

\begin{tabular}{llll}
\hline Infection condition & Differentially expressed genes $(+/-\geq$ 1folds, $\mathbf{p}<\mathbf{0 . 0 5})$ & Up-regulated genes & Down-regulated genes \\
\hline HPAIV H5N1 & 5550 & 4785 & 765 \\
LPAIV H9N2 & 2992 & 2552 & 440 \\
\hline
\end{tabular}

doi:10.1371/journal.pone.0153671.t001 


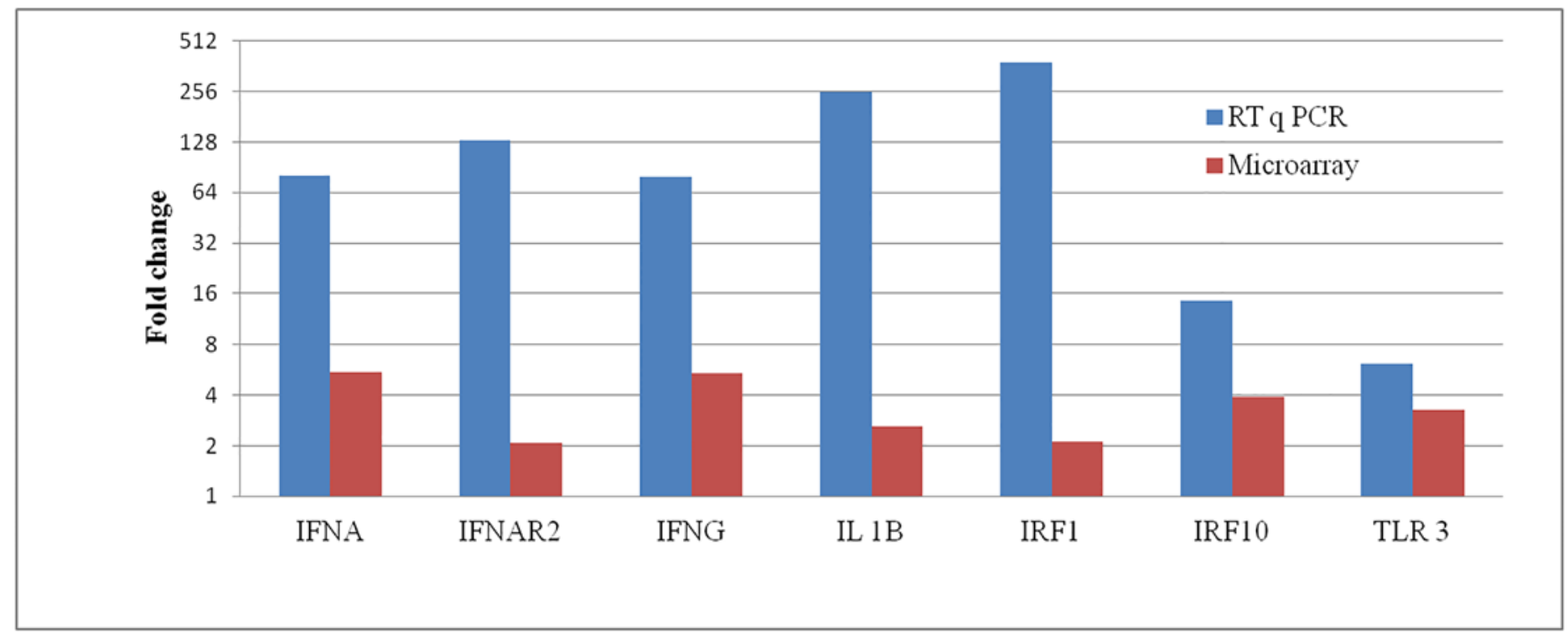

Fig 1. Validation of microarray data by RT qPCR. Relative amount of differentially expressed genes in HPAIV H5N1 infected as compared to non infected lung tissues using RT qPCR.

doi:10.1371/journal.pone.0153671.g001

were reported previously $[27,28]$. This general discrepancy in gene expression fold change values in microarray and RT qPCR techniques has been the subject of previous research [29, 30].

Gene ontology analysis of the upregulated genes revealed that the genes were involved in response to cytokine stimulus, inflammatory response, innate immunity, response to virus, regulation of adaptive immune response, and apoptosis (Table 2). Gene ontology analysis of downregulated genes revealed that the genes were involved in DNA repair, cellular response to stress, negative regulation of cellular biosynthetic process, cell cycle, positive regulation of signal transduction and transcription activator activity (Table 2). KEGG pathway analysis of HPAI H5N1 upregulated genes revealed enrichment of Influenza A pathway, Jak-STAT signaling pathway, NOD-like receptor signaling pathway, p53 signaling pathway, RIG-I-like receptor signaling pathway, Toll-like receptor signaling pathway, TNF signaling pathway etc. (Fig 2). HPAI H5N1 downregulated genes enriched the pathways such as Cytokine-cytokine receptor interaction, Jak-STAT signaling pathway, MAPK signaling pathway, PI3K-Akt signaling pathway etc. (Fig 2). However, the number of genes involved in particular pathway was quantitatively different between these up or down regulated genes list.

\section{Host gene response to LPAI H9N2 virus infection}

In order to determine the genes expressed in lung tissue in response to LPAI H9N2 virus, the infected tissues were compared to control tissues. Hemagglutinin titer of the LPAI H9N2 virus (A/duck/India/249800/2010) in infected lung tissues was $2^{8}$. In LPAIV infected lungs tissues, approximately 2992 genes were significantly differentially expressed at the cut-off of $\mathrm{p}$ value $\leq 0.05$ and fold change $\geq 1$ ( $\log 2$ transformed value) as against the control tissue. Of these, 2552 genes were upregulated and 440 genes were downregulated (Table 1). The immune genes upregulated in infected tissue include IFNA3, TRIM65, TRIM71, TGFA, IL13, IL12B, IL9A, IL23A, and other housekeeping genes. In LPAIV infected tissue IFITM5, TRIM50, TGFB3, PRLR, OXSR1 etc. genes were downregulated. The RT qPCR result shows moderate up regulation of IFNA, IFNG, IFNAR2, STAT3, Mx1, ILIB, TLR3, IRF1, and IRF10 genes during LPAIV infection in chicken (S1 Fig). 
The following gene ontology were enriched in upregulated genes including negative regulation of transcription, cell-cell signaling, response to wounding, $\mathrm{T}$ cell differentiation, positive regulation of lymphocyte activation, fatty acid metabolic process and response to carbohydrate stimulus (Table 3). The GO term enriched by the downregulated genes included cellular homeostasis, calcium ion homeostasis, apoptosis and microtubule-based movement (Table 3). The genes expressed in cellular homeostasis included ATP2A2, TXN2, RHOT2, PLN, DMD, APP, and Bak1. The genes upregulated in LPAIV H9N2 infected tissues are components of the Influenza A pathway, Jak-STAT signaling pathway, MAPK signaling pathway, NF-kappa B signaling pathway, RIG-I-like receptor signaling pathway, TNF signaling pathway and Toll-like receptor signaling pathway (Fig 3). The downregulated genes are components of the B cell receptor signaling pathway, Cytokine-cytokine receptor interaction, Fc gamma R-mediated phagocytosis, Jak-STAT signaling pathway, and MAPK signaling pathway (Fig 3).

\section{Discussion}

In this study, we examined the host responses to a highly-pathogenic H5N1 virus and a low pathogenic H9N2 virus infection in chickens. The differences in viral pathogenicity between HPAIV and LPAIV infection were related to the difference in host immune responses to these viruses.

\section{Immune response to HPAI H5N1 virus}

The influenza viral pathogen-associated molecular patterns (PAMPs) is recognized by host pattern recognition receptors (PRRs) such as Toll-like receptors TLR3 [31] and TLR7 [32],

Table 2. Significantly enriched Gene Ontology terms in response to HPAIV H5N1 infection.

\begin{tabular}{lll}
\hline GO Terms & Gene Count & p-value \\
\hline Upregulated genes & & \\
Positive regulation of immune system process & 42 & $1.10 \mathrm{E}-05$ \\
\hline Positive regulation of lymphocyte activation & 23 & $1.80 \mathrm{E}-05$ \\
Cytokine receptor activity & 14 & $7.00 \mathrm{E}-04$ \\
Response to virus & 21 & $8.60 \mathrm{E}-04$ \\
Inflammatory response & 45 & $1.60 \mathrm{E}-03$ \\
\hline Innate immunity & 15 & $2.10 \mathrm{E}-03$ \\
Regulation of protein kinase cascade & 36 & $2.40 \mathrm{E}-03$ \\
Negative regulation of biosynthetic process & 69 & $3.60 \mathrm{E}-03$ \\
Regulation of interleukin-2 production & 8 & $1.20 \mathrm{E}-02$ \\
Regulation of apoptosis & 88 & $1.30 \mathrm{E}-02$ \\
Positive regulation of chemokine biosynthetic process & 4 & $1.70 \mathrm{E}-02$ \\
Antigen processing and presentation of endogenous antigen & 4 & $2.50 \mathrm{E}-02$ \\
Positive regulation of signal transduction & 41 & $2.40 \mathrm{E}-03$ \\
Response to wounding & 78 & $2.50 \mathrm{E}-06$ \\
\hline Transcription factor activity & 143 & $2.60 \mathrm{E}-10$ \\
\hline Positive regulation of gene expression & 88 & $1.40 \mathrm{E}-07$ \\
Regulation of interleukin-6 biosynthetic process & 5 & $2.70 \mathrm{E}-02$ \\
Downregulated genes & & $1.50 \mathrm{E}-03$ \\
Response to DNA damage stimulus & 15 & $5.10 \mathrm{E}-03$ \\
Cellular response to stress & 18 & $2.20 \mathrm{E}-02$ \\
Negative regulation of cellular biosynthetic process & 16 & $4.40 \mathrm{E}-02$ \\
\hline Cell cycle & 19 & $4.70 \mathrm{E}-02$ \\
\hline Transcription activator activity & 12 & $4.90 \mathrm{E}-02$ \\
\hline Negative regulation of nitrogen compound metabolic process & 14 & \\
\hline & & \\
\hline
\end{tabular}

doi:10.1371/journal.pone.0153671.t002 


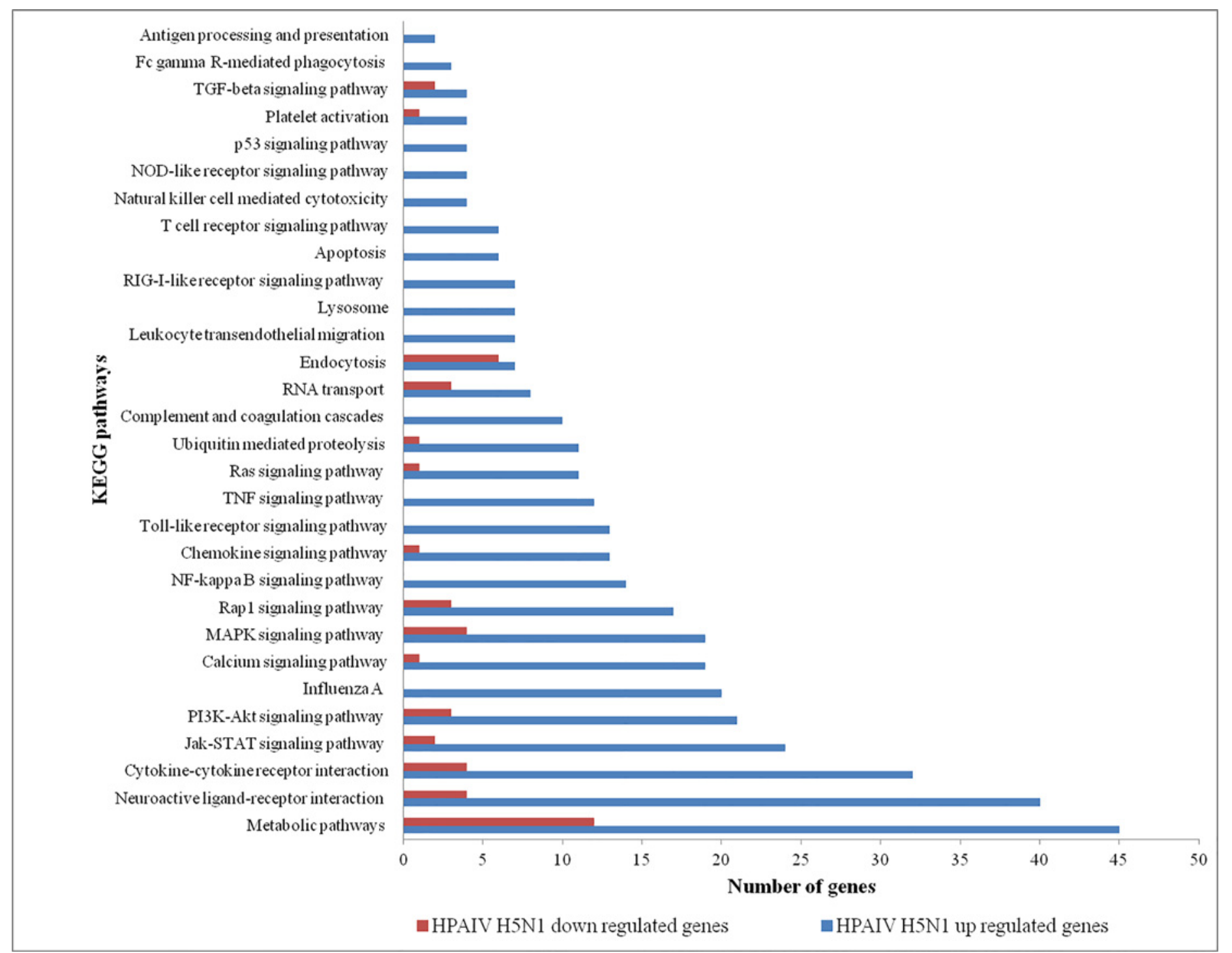

Fig 2. KEGG pathways enriched in response to HPAIV H5N1 infection. Up regulated and down regulated genes differentially enriched in various pathways are shown.

doi:10.1371/journal.pone.0153671.g002

retinoic acid-inducible gene I (RIG-I) [33] and the NOD-like receptor family member NOD-, LRR- and pyrin domain-containing 3 (NLRP3/NLRC5) [34], and activation of these PRRs induces secretion of type I interferons (IFNs), pro-inflammatory cytokines and chemokines [35]. In HPAIV infected chicken, MDA5 (IFIH1), TLR3, and NLRC5 genes were upregulated in lung tissues with fold change value of $3.7,3.3$, and 2.9 respectively.

TLRs triggers the activation of transcription factors [nuclear factor- $\kappa \mathrm{B}(\mathrm{NF}-\kappa \mathrm{B})$ or IFN-regulatory factor 7 (IRF7)] responsible for the expression of type I IFNs and proinflammatory cytokines [36, 37]. In this study, TLR15 (fold change value 2.7) and TLR6 (fold change value 2.2) genes were found to be upregulated in HPAIV infected tissues. Further, MYD88 and IRF7 genes were upregulated and NF- $\mathrm{KB}$ signaling pathway was enriched (Fig 2). The up regulation of IFNA and IFNAR2 genes showed fold change value of 5.5 and 2.0 respectively in HPAIV infected tissues and may have been stimulated by NF- $\kappa \mathrm{B}$ signaling pathway. 
Table 3. Significantly enriched Gene Ontology terms in response to LPAIV H9N2 infection.

\begin{tabular}{lll}
\hline GO Terms & Gene Count & p-value \\
\hline Upregulated genes & & \\
Negative regulation of RNA metabolic process & 30 & $2.10 \mathrm{E}-04$ \\
Negative regulation of transcription & 35 & $2.70 \mathrm{E}-04$ \\
Cell -cell signaling & 41 & $6.90 \mathrm{E}-04$ \\
Negative regulation of macromolecule biosynthetic process & 38 & $8.40 \mathrm{E}-04$ \\
Response to wounding & 36 & $1.70 \mathrm{E}-03$ \\
Response to abiotic stimulus & 27 & $2.70 \mathrm{E}-03$ \\
Positive regulation of homeostatic process & 7 & $4.30 \mathrm{E}-03$ \\
Regulation of cAMP metabolic process & 10 & $1.90 \mathrm{E}-02$ \\
Positive regulation of lymphocyte activation & 9 & $3.60 \mathrm{E}-02$ \\
\hline T cell differentiation & 7 & $4.10 \mathrm{E}-02$ \\
Cell -cell adhesion & 18 & $4.40 \mathrm{E}-02$ \\
Regulation of nucleotide metabolic process & 10 & $3.80 \mathrm{E}-02$ \\
Fatty acid metabolic process & 16 & $1.10 \mathrm{E}-02$ \\
Response to carbohydrate stimulus & 7 & $2.90 \mathrm{E}-02$ \\
\hline Downregulated genes & & \\
cellular homeostasis & 7 & $2.30 \mathrm{E}-03$ \\
Apoptosis & 5 & $2.00 \mathrm{E}-02$ \\
Cytoskeleton -dependent intracellular transport & 3 & $1.20 \mathrm{E}-02$ \\
Microtubule -based movement & 4 & $1.70 \mathrm{E}-02$ \\
Calcium ion homeostasis & 4 & $5.00 \mathrm{E}-03$ \\
\hline
\end{tabular}

doi:10.1371/journal.pone.0153671.t003

In the lack of RIG-I gene in chicken, MDA5 (IFIH1) gene functionally compensate the immune functions of RIG-I $[38,39]$. Chicken MDA5 receptor, which uses the same signaling pathway as RIG-I, results in induction of type I IFNs and involves chicken LGP2, MAVS and IRF3/IRF7 genes [25, 40, 41]. In HPAIV infected tissues IFIH1 and IRF7 genes were upregulated indicating the activation of the type I IFN (IFNA) response in lungs. Other IFN genes namely IFNG and IFNL as well as interferon regulatory factor namely IRF1, IRF10, IRF2 and IRF8 were also upregulated in HPAIV infected tissues. Increased expressions of these genes were confirmed by RT qPCR (Fig 1). Significant increases in IFNA, IFNG and TLR3 mRNA expression are reported during HPAI infection of chicken dendritic cells [42]. The type I and III IFNs expressed through different PRRs bind to the IFNAR1/IFNAR2 and IFNGR1 and IFNGR2 receptors respectively. This receptor complex activate Jak-STAT signaling through a trimeric transcription factor complex (ISGF3) results in induction of range of IFN stimulated genes (ISGs) [43].

Amongst the IFN-stimulated genes, we found more genes were upregulated in response to H5N1 infection including the MX1, OASL, IFITM5 and IFITM3, IFIT5, EIF2AK1 (PKR) and EIF2AK2, GBP 1 and 7, SLC16A1, FAM46A, RSAD2, and ZC3HAV1 (Fig 4). Similarly, we found that a number of IFN-stimulated pro-inflammatory cytokines (IL1B, IL10RA, IL10RB, IL12B, IL13, IL13RA1, IL13RA2, IL15RA, IL17F, IL17RA, IL18, IL18R1, IL22, IL22RA2, IL23A, IL28B, IL2RA, IL4I1, IL6ST and IL9R) and the chemokines (CCL4, CCL19, CCL10, CXCL12 and CX3CL) were also upregulated in HPAIV infected lung tissues of chicken (Fig 4). The RT qPCR analysis confirmed increased ILIB, IRF1 and MX gene expression in the HPAI virus-infected lung tissues (Fig 1). Several of these genes, including MX, OAS, PKR, IFITM and IFIT5 expression have been implicated in influenza virus infection. MX gene is first reported ISGs that restrict influenza virus infection $[44,45]$. IFITM proteins restrict the influenza A 


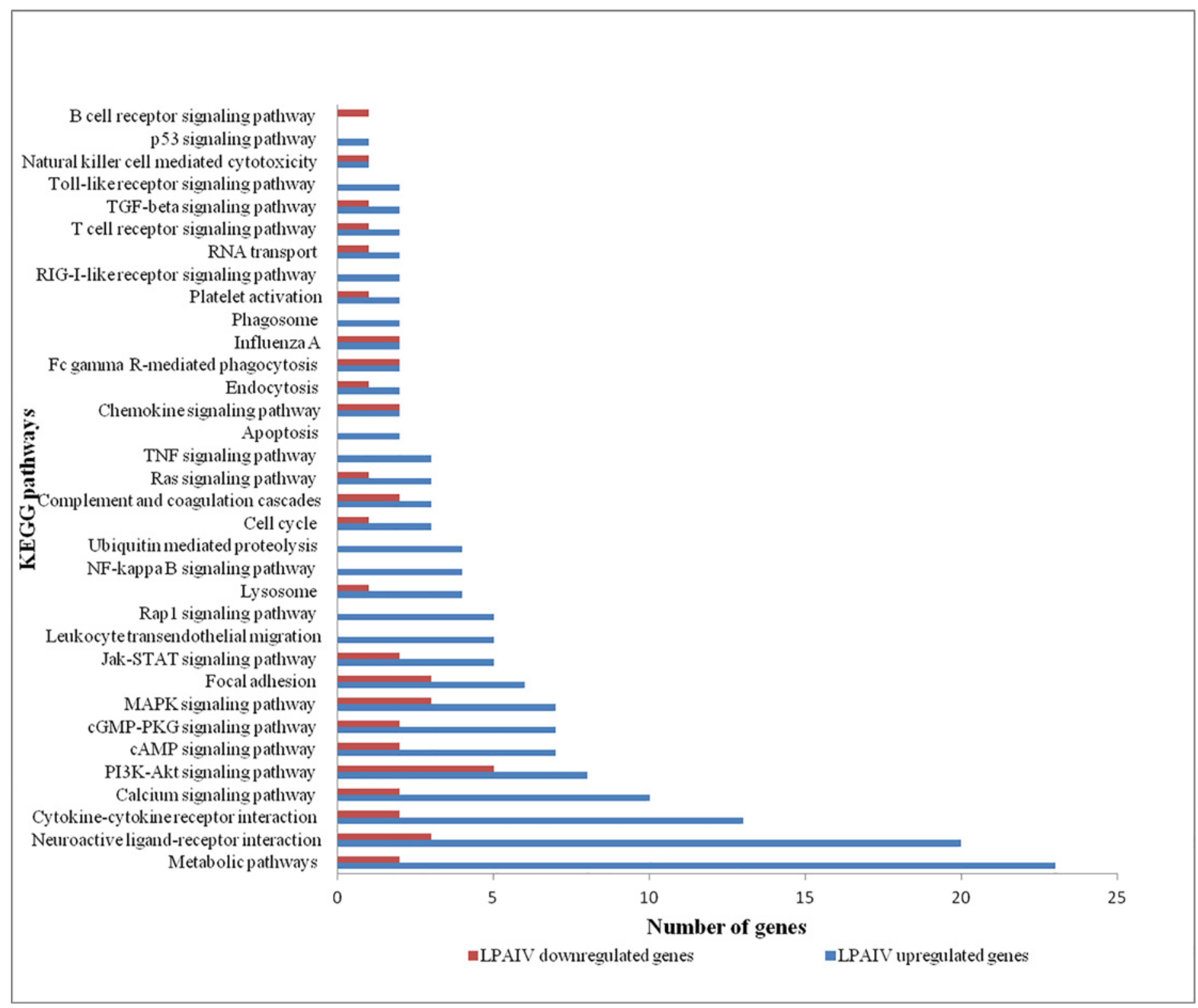

Fig 3. KEGG pathways enriched in response to LPAIV H9N2 infection. Up regulated and down regulated genes differentially enriched in various pathways are shown.

doi:10.1371/journal.pone.0153671.g003

virus replication $[46,47]$. The OAS family and RNase L act together to degrade viral RNA in the cytosol [48]. PKR protein restricts viral replication by inhibition of translation of viral mRNA $[49,50]$. The IFIT family proteins recognize viral RNA with 5 'triphosphate thereby IFIT complex antagonizes viruses by sequestering specific viral nucleic acids [51].

In spite of the fact that the host is induced to express a good number of IFN-stimulated genes and pro-inflammatory cytokines, influenza virus evade the host's immune response and efficiently replicate in lung tissues. The influenza virus evade host's immune response by induction of suppressor of cytokine signaling (SOCS), a potent endogenous inhibitor of TLR signaling and IFN signaling [52-56]. SOCS1 (fold change value 3.7), SOCS3 (fold change value 4.2), SOCS4 (fold change value 1.4), and SOCS5 (fold change value 2.3) genes were 


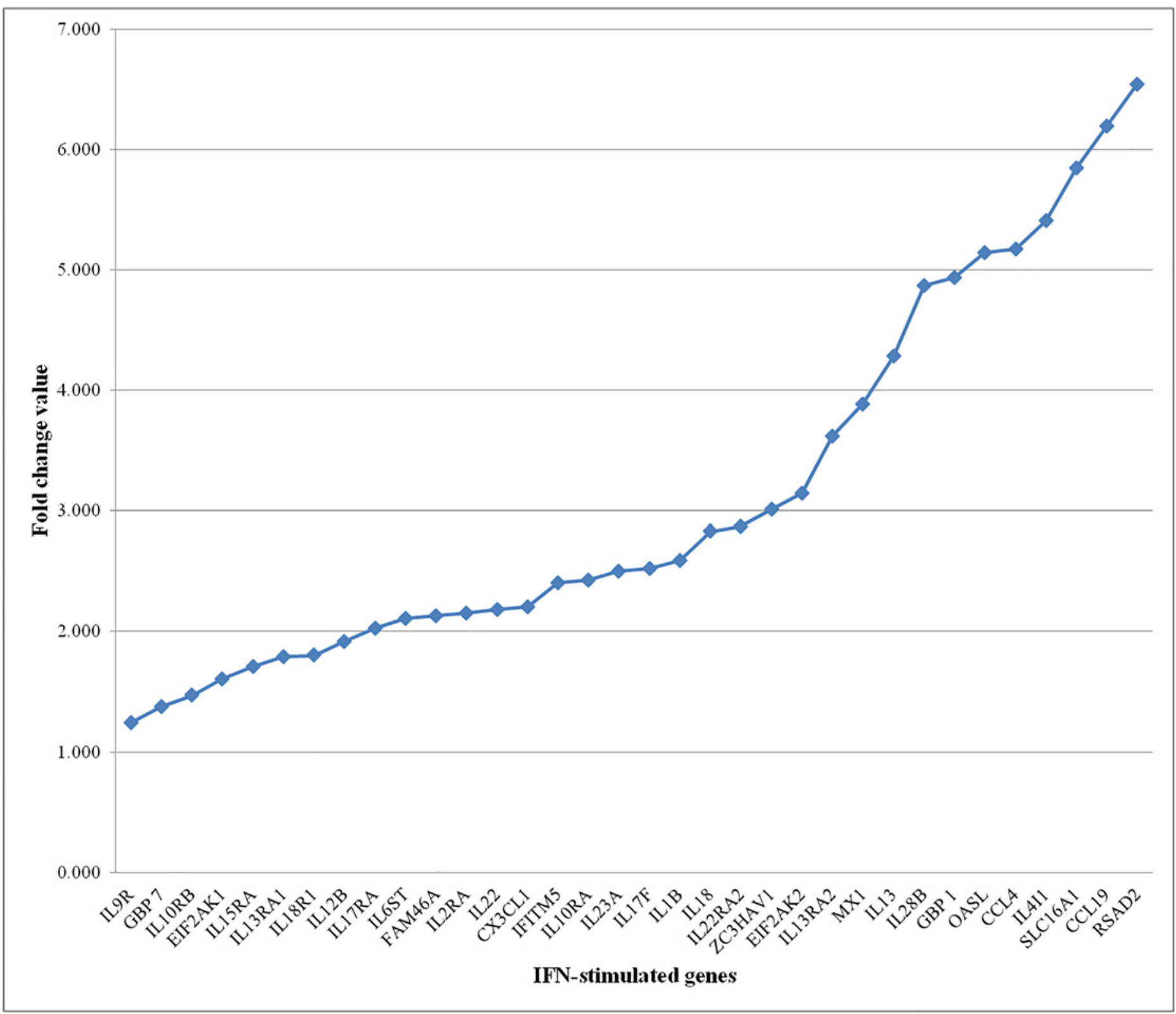

Fig 4. IFN-stimulated genes were expressed in response to HPAIV H5N1 infection in chicken lung tissues. The IFN-stimulated genes, proinflammatory cytokines and chemokines were all upregulated in HPAIV H5N1 infected lung tissues of chicken.

doi:10.1371/journal.pone.0153671.g004

upregulated in HPAIV H5N1 infected lung tissues. Upregulation of SOCS genes inhibit the antiviral response of the host and pave the path for efficient virus replication in the lung tissues [27]. This increased viral load in the lung tissues induces excessive proinflammatory cytokines expressions [27]. Further activation of TNF signaling pathways induce increased expression levels of many pro-inflammatory cytokines [27]. TNF signaling pathway genes (CEBPB, CX3CL1, IL18R1, IL1B, MAP3K14, MMP14, PTGS2, SOCS3, TNFAIP3, TRAF2, TRAF3, VCAM1) and TNF super family genes (TNFAIP3, TNFAIP6, TNFRSF10B, TNFRSF13C, TNFRSF21, TNFSF13B, TNFSF15 and TNFSF8) were upregulated in HPAIV infected tissues. 
Higher level expression of proinflammatory cytokines and chemokines, including IFNA, IFNG, TLRs, IL-1, IL-6, IL-12, IL-10, IL-18, TNF- $\alpha$, CCL2, CCL4, CCL5, and CXCL10 were reported in humans and animal models infected with pandemic /H5N1 influenza viruses [10, 11,13-16]. Similarly the HPAI H5N1 infection resulted in very high transcriptional induction of IFNA $[5,57,58]$, IFNG $[5,59]$, IL1B $[5,59]$, and IL6 $[5,57,59,60]$ was reported in chickens using RT-qPCR. IL-6 expression is directly linked to host morbidity [61, 62]. Chemokines expression at influenza virus infections correlates with disease severity and mortality $[10,15$, 63]. This intense host inflammatory immune response cause permanent lung tissues damage result in critical organ failure and death of the host $[5,59,60]$. In summary, the present genome wide host gene expression study clearly indicates that the $\mathrm{H} 5 \mathrm{~N} 1$ virus induces hypercytokine responses resulted in high mortality in chickens.

Avian influenza virus replication in cells leads to the induction of apoptotic cell death and apoptosis has been suggested to contribute to the mortality of the host [2]. Activation of apoptosis pathways such as p53 signaling pathway, MAPK signaling pathway and PI3K-Akt signaling pathway were observed in HPAIV infected lung tissues (Fig 2). The expression of genes

MAPK12, MAPK1IP1L, MAPK8IP3 and MAPKAP1 that are associated with cell death were also observed in $\mathrm{H} 5 \mathrm{~N} 1$ virus-infected tissues. Watanabe et al reported 128 human genes involved in influenza virus replication cycle [64]. Some of these genes such as RPS4X, GRK6, RUNX1, PHF2, STARD5, IRF2, IL17RA, APC2, and IFIT5 etc. were expressed in our transcriptome data (Fig 5).

\section{Immune response to LPAI H9N2 virus}

Most low pathogenic avian influenza viruses cause no or mild disease in avian species. Little is known about immune response and molecular mechanism of tolerance to LPAIVs infection in birds [6]. To characterize the transcriptional immune response to LPAI H9N2 virus, we analyzed the differentially regulated genes in chicken lung tissues using microarray. Infection of chicken with LPAIV induces high levels of IFN in the lung tissues $[28,58,65]$. However in our case LPAIV H9N2 virus induced the expression of type I interferon (IFNA) with fold change value of 1.8 in lung tissues. Xing et al suggested that weak induction of IFNs in H9N2 infected macrophages may probably contributed to differential regulation of IFN-inducible genes [6]. Among the differentially regulated genes, IFITM5, TRIM50, and TGFB3 genes were downregulated in the lung tissues. Additionally, interleukins like IL12B, IL13, IL17F, IL23A, IL9A, and TGFA were all upregulated in LPAIV H9N2 infected lung tissues. The expression of other interleukins and chemokines genes were not induced in the lungs tissues of chickens infected with LPAI H9N2 virus.

The immune pathways activated in response to LPAIV infection in chickens were the TLR pathway, RIG-I pathway, Jak-STAT signaling pathway, NF-kappa B signaling pathway, Cytokine-cytokine receptor interaction, MAPK signaling pathway, TGF-beta signaling pathway, Chemokine signaling pathway etc. (Fig 3). Activation of these pathways in chicken lungs confirmed an innate immune response at the transcriptional level. Many of these immune pathways were previously reported to associate with influenza virus infection [2, 27, 56, 66, 67]. Maughan et al reported that ducks are able to tolerate negative consequences associated with LPAIV infection through fine-tune their innate immune response by differentially regulating the various immune related pathways [66]. Similarly, chickens may differently regulate these immune pathways to tolerate LPAIV infections.

In conclusion, highly pathogenic $\mathrm{H} 5 \mathrm{~N} 1$ virus induced excessive expression of innate immune genes in the lung tissues. This atypical expression of immune genes might be the cause for the high mortality in chickens. In contrast, the expression of most of these genes was either not induced or some were downregulated except for a modest expression of some immune genes 


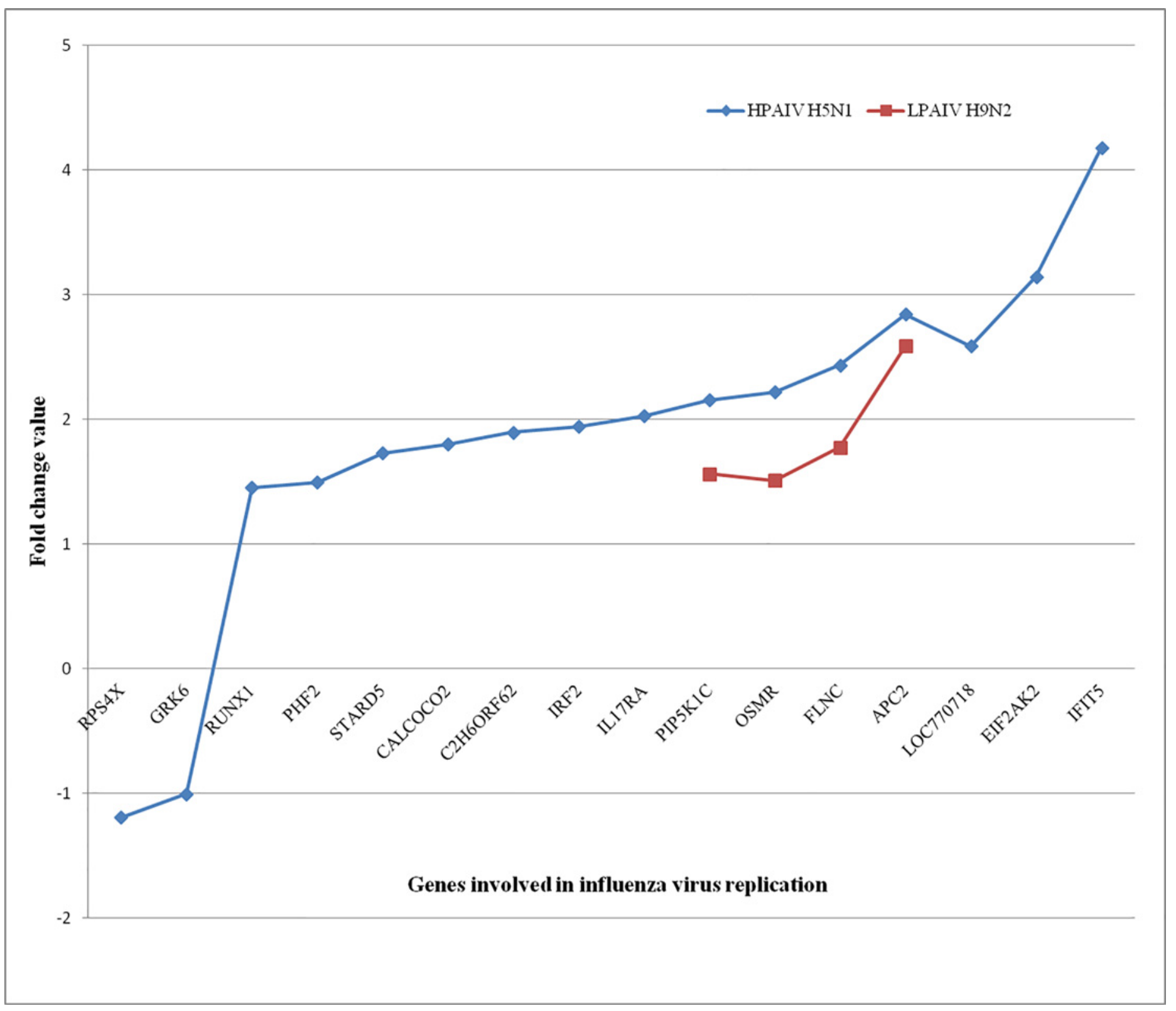

Fig 5. The host genes were involved in avian influenza virus replication in chicken lung tissues are shown along with fold change expression value.

doi:10.1371/journal.pone.0153671.g005

such as IFNA, IFITM5, TGFB3, IL12B, IL13, IL17F were observed in LPAI H9N2 virus infection in chickens. Hence further studies are required to elucidate what host factors are controlling the gene expression during infection to recover or tolerate LPAIV infection in chickens.

\section{Supporting Information}

S1 Fig. Validation of microarray data by RT qPCR. Relative amount of differentially expressed genes in LPAIV H5N1 infected as compared to non infected lung tissues using RT qPCR.

(TIFF) 


\section{Acknowledgments}

We thank Director, ICAR-National Institute of High Security Animal Diseases, Director, ICAR-Indian Veterinary Research Institute, and Indian Council of Agricultural Research, India for providing necessary facilities to carry out this work.

\section{Author Contributions}

Conceived and designed the experiments: AAR AM DDK. Performed the experiments: AAR AM PBR PV. Analyzed the data: AM PBR PV HK. Contributed reagents/materials/analysis tools: PNG. Wrote the paper: PV AM.

\section{References}

1. OIE. Avian influenza. Terrestrial animal health code. World Organisation for Animal Health, Paris, 2009. pp 1-20.

2. Post J, Burt DW, Cornelissen JB, Broks V, van Zoelen D, Peeters B et al. Systemic virus distribution and host responses in brain and intestine of chickens infected with low pathogenic or high pathogenic avian influenza virus. Virol J. 2012; 9:61. doi: 10.1186/1743-422X-9-61 PMID: 22390870

3. Pantin-Jackwood MJ, Swayne DE. Pathogenesis and pathobiology of avian influenza virus infection in birds. Rev Sci Tech. 2009; 28:113-36. PMID: 19618622

4. Steinhauer DA. Role of hemagglutinin cleavage for the pathogenicity of influenza virus. Virology. 1999; 258:1-20. PMID: 10329563

5. Kuribayashi S, Sakoda Y, Kawasaki T, Tanaka T, Yamamoto $\mathrm{N}$ et al. Excessive Cytokine Response to Rapid Proliferation of Highly Pathogenic Avian Influenza Viruses Leads to Fatal Systemic Capillary Leakage in Chickens. PLoS ONE. 2013: 8: e68375 doi: 10.1371/journal.pone.0068375 PMID: 23874602

6. Xing Z, Cardona CJ, Li J, Dao N, Tran T, Andrada J. Modulation of the immune responses in chickens by low-pathogenicity avian influenza virus H9N2. J Gen Virol. 2008; 89:1288-99. doi: 10.1099/vir.0. 83362-0 PMID: 18420808

7. Alexander DJ. A review of avian influenza in different bird species. Vet Microbio. 2000; 174: 3-13.

8. Guo Y, Li J, Cheng X. Discovery of humans infected by avian influenza A (H9N2) virus. Zhonghua Shi Yan He Lin Chuang Bing Du Xue Za Zhi. 1999; 13:105-108. Chinese. PMID: 12569771

9. Peiris $M$, Yuen $K Y$, Leung $C W$, Chan $K H$, Ip PL, Lai RW et al. Human infection with influenza H9N2. Lancet. 1999; 354:916-917. PMID: 10489954

10. de Jong MD, Simmons CP, Thanh TT, Hien VM, Smith GJ, Chau TN et al. Fatal outcome of human influenza a (H5N1) is associated with high viral load and Hypercytokinemia. Nat Med. 2006; 12:12037. PMID: 16964257

11. La Gruta NL, Kedzierska K, Stambas J, Doherty PC. A question of self-preservation: immunopathology in influenza virus infection. Immunol. Cell Biol. 2007; 85(2):85-92. Epub 2007 Jan 9. PMID: 17213831

12. Lipatov AS, Andreansky S, Webby RJ, Hulse DJ, Rehg JE, Krauss S et al. Pathogenesis of Hong Kong H5N1 influenza virus NS gene reassortants in mice: the role of cytokines and B- and T-cell responses. J Gen Virol. 2005; 86:1121-1130. PMID: 15784906

13. Szretter KJ, Gangappa S, Lu X, Smith C, Shieh WJ, Zaki SR et al. Role of host cytokine responses in the pathogenesis of avian H5N1 influenza viruses in mice. J Virol. 2007; 81:2736-2744. PMID: 17182684

14. Cameron CM, Cameron MJ, Bermejo-Martin JF, Ran L, Xu L, Turner PV et al. Gene Expression Analysis of Host Innate Immune Responses during Lethal H5N1 Infection in Ferrets. J Virol. 2008; 82:1130811317. doi: 10.1128/JVI.00691-08 PMID: 18684821

15. Kobasa D, Jones SM, Shinya K, Kash JC, Copps J, Ebihara $\mathrm{H}$ et al. Aberrant innate immune response in lethal infection of macaques with the 1918 influenza virus. Nature. 2007; 445:319-23. PMID: 17230189

16. Cillo'niz C, Shinya K, Peng X, Korth MJ, Proll SC, Aicher LD et al. Lethal Influenza Virus Infection in Macaques Is Associated with Early Dysregulation of Inflammatory Related Genes. PLoS Pathog. 2009; 5(10): e1000604. doi: 10.1371/journal.ppat.1000604 PMID: 19798428

17. Chan MC, Cheung CY, Chui WH, Tsao SW, Nicholls JM, Chan YO et al. Proinflammatory cytokine responses induced by influenza $A(\mathrm{H} 5 \mathrm{~N} 1)$ viruses in primary human alveolar and bronchial epithelial cells. Respir Res. 2005; 6:135. PMID: 16283933 
18. Suzuki K, Okada H, Itoh $\mathrm{T}$, Tada $\mathrm{T}$, Mase M, Nakamura K et al. Association of increased pathogenicity of Asian $\mathrm{H} 5 \mathrm{~N} 1$ highly pathogenic avian influenza viruses in chickens with highly efficient viral replication accompanied by early destruction of innate immune responses. J Virol. 2009; 83:7475-7486. doi: 10. 1128/JVI.01434-08 PMID: 19457987

19. Reemers SS, van Haarlem DA, Groot Koerkamp MJ, Vervelde L. Differential gene-expression and host-response profiles against avian influenza virus within the chicken lung due to anatomy and airflow. J Gen Virol. 2009; 90:2134-2146. doi: 10.1099/vir.0.012401-0 PMID: 19494054

20. Rebel JM, Peeters B, Fijten H, Post J, Cornelissen J, Vervelde L. Highly pathogenic or low pathogenic avian influenza virus subtype H7N1 infection in chicken lungs: small differences in general acute responses. Vet Res. 2011; 42:10. doi: 10.1186/1297-9716-42-10 PMID: 21314972

21. Hale BG, Albrecht RA, García-Sastre A. Innate immune evasion strategies of influenza viruses. Future Microbiol. 2010; 5: 23. doi: 10.2217/fmb.09.108 PMID: 20020828

22. Webster RG, Bean WJ, Gorman OT, Chambers TM, Kawaoka Y. Evolution and ecology of influenza A viruses. Microbiol Rev. 1992; 56:152-179. PMID: 1579108

23. Li YP, Handberg KJ, Juul-Madsen HR, Zhang MF, Jørgensen PH. Transcriptional profiles of chicken embryo cell cultures following infection with infectious bursal disease virus. Arch Virol. 2007; 152 (3):463-78. Epub 2006 Dec 4. PMID: 17143781

24. Nang NT, Lee JS, Song BM, Kang YM, Kim HS, Seo SH. Induction of inflammatory cytokines and tolllike receptors in chickens infected with avian H9N2 influenza virus. Vet Res. 2011; 42:64. doi: 10.1186/ 1297-9716-42-64 PMID: 21592354

25. Barber MR, Aldridge JR Jr, Fleming-Canepa X, Wang YD, Webster RG, Magor KE. Identification of avian RIG-I responsive genes during influenza infection. Mol Immunol. 2013; 54:89-97. doi: 10.1016/j. molimm.2012.10.038 PMID: 23220072

26. Schmittgen TD1, Livak KJ. Analyzing real-time PCR data by the comparative $C(T)$ method.Nat Protoc. 2008; 3(6):1101-8. PMID: 18546601

27. Lee SMY, Gardy JL, Cheung CY, Cheung TKW, Hui KPY, Ip NY et al. Systems-Level Comparison of Host-Responses Elicited by Avian H5N1 and Seasonal H1N1 Influenza Viruses in Primary Human Macrophages. PLoS ONE. 2009; 4(12): e8072. doi: 10.1371/journal.pone.0008072 PMID: 20011590

28. Sutejo R, Yeo DS, Myaing MZ, Hui C, Xia J, Ko D et al. Activation of Type I and III Interferon Signalling Pathways Occurs in Lung Epithelial Cells Infected with Low Pathogenic Avian Influenza Viruses. PLoS ONE. 2012; 7(3): e33732. doi: 10.1371/journal.pone.0033732 PMID: 22470468

29. Yuen T, Wurmbach E, Pfeffer RL, Ebersole BJ, Sealfon SC. Accuracy and calibration of commercial oligonucleotide and custom cDNA microarrays. Nucleic Acids Res. 2002; 30: e48. PMID: 12000853

30. Dallas PB, Gottardo NG, Firth MJ, Beesley AH, Hoffmann K, Terry PA et al. Gene expression levels assessed by oligonucleotide microarray analysis and quantitative real-time RT-PCR-how well do they correlate? BMC Genomics. 2005; 6: 59. PMID: 15854232

31. Le Goffic R, Pothlichet J, Vitour D, Fujita T, Meurs E, Chignard M et al. Cutting Edge: Influenza A virus activates TLR3-dependent inflammatory and RIG-I-dependent antiviral responses in human lung epithelial cells. J Immunol. 2007; 78:3368-3372.

32. Lund JM, Alexopoulou L, Sato A, Karow M, Adams NC, Gale NW et al. Recognition of single-stranded RNA viruses by Toll-like receptor 7. Proc. Natl Acad. Sci. USA. 2004; 101:5598-5603. PMID: 15034168

33. Pichlmair A, Schulz O, Tan CP, Näslund TI, Liljeström P, Weber F et al. RIG-I-mediated antiviral responses to single-stranded RNA bearing 5'-phosphates. Science. 2006; 314:997-1001. PMID: 17038589

34. Pothlichet J, Meunier I, Davis BK, Ting JP, Skamene E, von Messling V et al. Type I IFN triggers RIG-I/ TLR3/NLRP3-dependent inflammasome activation in influenza A virus infected cells. PLoS Pathog. 2013; 9:e1003256. doi: 10.1371/journal.ppat.1003256 PMID: 23592984

35. Iwasaki A, Pillai PS. Innate immunity to influenza virus infection. Nat Rev Immunol. 2014; 14(5):31528. doi: 10.1038/nri3665 PMID: 24762827

36. Honda K, Ohba Y, Yanai H, Negishi H, Mizutani T, Takaoka A et al. Spatiotemporal regulation of MyD88-IRF-7 signalling for robust type-I interferon induction. Nature. 2005; 434:1035-1040. PMID: 15815647

37. Sasai M, Linehan MM, Iwasaki A. Bifurcation of Toll-like receptor 9 signaling by adaptor protein 3 . Science. 2010; 329:1530-1534. doi: 10.1126/science.1187029 PMID: 20847273

38. Barber MR, Aldridge JR Jr, Webster RG, Magor KE. Association of RIG-I with innate immunity of ducks to influenza. Proc Natl Acad Sci USA. 2010; 107:5913-5918. doi: 10.1073/pnas.1001755107 PMID: 20308570 
39. Karpala AJ, Stewart C, McKay J, Lowenthal JW, Bean AG. Characterization of chicken Mda5 activity: regulation of IFN- $\beta$ in the absence of RIG-I functionality. J Immunol. 2011; 186(9):5397-405. doi: 10. 4049/jimmunol.1003712 Epub 2011 Mar 28. PMID: 21444763

40. Grant CE, Vasa MZ, Deeley RG. cIRF-3, a new member of the interferon regulatory factor (IRF) family that is rapidly and transiently induced by dsRNA. Nucleic Acids Res. 1995; 23:2137-2146. PMID: 7541908

41. Liniger M, Summerfield A, Zimmer G, McCullough KC, Ruggli N. Chicken cells sense influenza A virus infection through MDA5 and CARDIF signaling involving LGP2. J Virol. 2012; 86:705-717. doi: 10. 1128/JVI.00742-11 PMID: 22072756

42. Vervelde L, Reemers SS, van Haarlem DA, Post J, Claassen E, Rebel JM et al. Chicken dendritic cells are susceptible to highly pathogenic avian influenza viruses which induce strong cytokine responses. Dev Comp Immunol. 2013; 39(3):198-206. doi: 10.1016/j.dci.2012.10.011 Epub 2012 Nov 23. PMID: 23178410

43. Randall RE, Goodbourn S. Interferons and viruses: An interplay between induction, signalling, antiviral responses and virus countermeasures. J Gen Virol. 2008; 89:1-47. PMID: 18089727

44. Staeheli $\mathrm{P}$, Haller O, Boll W, Lindenmann J, Weissmann C. Mx protein: constitutive expression in $3 T 3$ cells transformed with cloned Mx cDNA confers selective resistance to influenza virus. Cell. 1986; 44:147-158. PMID: 3000619

45. Turan K, Mibayashi M, Sugiyama K, Saito S, Numajiri A, Nagata K. Nuclear MxA proteins form a complex with influenza virus NP and inhibit the transcription of the engineered influenza virus genome. Nucleic Acids Res. 2004; 32:643-652. PMID: 14752052

46. Brass AL, Huang IC, Benita Y, John SP, Krishnan MN, Feeley EM et al. The IFITM proteins mediate celIular resistance to influenza A H1N1 virus, West Nile virus, and dengue virus. Cell. 2009; 139:12431254. doi: 10.1016/j.cell.2009.12.017 PMID: 20064371

47. Huang Y, Li Y, Burt DW, Chen $\mathrm{H}$, Zhang Y, Qian W et al. The duck genome and transcriptome provide insight into an avian influenza virus reservoir species. Nat Genet. 2013; 7:776-83.

48. Silverman $\mathrm{RH}$. Viral encounters with $2^{\prime}, 5^{\prime}$-oligoadenylate synthetase and $\mathrm{RNase} L$ during the interferon antiviral response. J Virol. 2007; 81:12720-12729. PMID: 17804500

49. Balachandran S, Roberts PC, Brown LE, Truong H, Pattnaik AK, Archer DR et al. Essential role for the dsRNA-dependent protein kinase PKR in innate immunity to viral infection. Immunity. 2000; 13:129141. PMID: 10933401

50. Dauber B, Martínez-Sobrido L, Schneider J, Hai R, Waibler Z, Kalinke U et al. Influenza B virus ribonucleoprotein is a potent activator of the antiviral kinase PKR. PLoS Pathog. 2009; 5(6):e1000473. doi: 10.1371/journal.ppat.1000473 Epub 2009 Jun 12. PMID: 19521506

51. Pichlmair A, Lassnig C, Eberle CA, Górna MW, Baumann CL, Burkard TR et al. IFIT1 is an antiviral protein that recognizes 5'-triphosphate RNA. Nat Immunol. 2011; 12(7):624-30. doi: 10.1038/ni.2048 PMID: 21642987

52. Gingras S, Parganas E, de Pauw A, Ihle JN, Murray PJ. Re-examination of the role of suppressor of cytokine signaling 1 (SOCS1) in the regulation of toll like receptor signaling. J Biol Chem. 2004; 279: 54702-54707. PMID: 15491990

53. Mansell A, Smith R, Doyle SL, Gray P, Fenner JE, Crack PJ et al. Suppressor of cytokine signaling 1 negatively regulates Toll-like receptor signaling by mediating Mal degradation. Nat Immunol. 2006; 7 : 148-155. PMID: 16415872

54. Pothlichet $\mathrm{J}$, Chignard $\mathrm{M}, \mathrm{Si}$-Tahar $\mathrm{M}$. Cutting edge: innate immune response triggered by influenza $\mathrm{A}$ virus is negatively regulated by SOCS1 and SOCS3 through a RIG-I/IFNAR1-dependent pathway. J Immunol. 2008; 180: 2034-2038. PMID: 18250407

55. Pauli E- K, Schmolke M, Wolff T, Viemann D, Roth J, Bode JG et al. Influenza A Virus Inhibits Type I IFN Signaling via NF-kB-Dependent Induction of SOCS-3 Expression. PLoS Pathog. 2008; 4(11): e1000196. doi: 10.1371/journal.ppat.1000196 PMID: 18989459

56. Vijayakumar P, Mishra A, Ranaware PB, Kolte AP, Kulkarni DD, Burt DW et al. Analysis of the crow lung transcriptome in response to infection with highly pathogenic $\mathrm{H} 5 \mathrm{~N} 1$ avian influenza virus. Gene. 2015; 559(1):77-85. doi: 10.1016/j.gene.2015.01.016 PMID: 25592823

57. Karpala AJ, Bingham J, Schat KA, Chen LM, Donis RO, Lowenthal JW et al. Highly pathogenic (H5N1) avian influenza induces an inflammatory T helper type 1 cytokine response in the chicken. $J$ Interferon Cytokine Res. 2011; 31(4):393-400. doi: 10.1089/jir.2010.0069 Epub 2011 Jan 1. PMID: 21194349

58. Moulin HR, Liniger M, Python S, Guzylack-Piriou L, Ocaña-Macchi M, Ruggli N et al. High interferon type I responses in the lung, plasma and spleen during highly pathogenic H5N1 infection of chicken. Vet Res. 2011; 42:6. doi: 10.1186/1297-9716-42-6 PMID: 21314963 
59. Cornelissen JB, Vervelde L, Post J, Rebel JM. Differences in highly pathogenic avian influenza viral pathogenesis and associated early inflammatory response in chickens and ducks. Avian Pathol. 2013; 42(4):347-64. doi: 10.1080/03079457.2013.807325 Epub 2013 Jun 19. PMID: 23782222

60. Burggraaf S, Karpala AJ, Bingham J, Lowther S, Selleck P, Kimpton W et al. H5N1 infection causes rapid mortality and high cytokine levels in chickens compared to ducks.Virus Res. 2014; 185:23-31. doi: 10.1016/j.virusres.2014.03.012 Epub 2014 Mar 19. PMID: 24657784

61. Kozak W, Poli V, Soszynski D, Conn CA, Leon LR, Kluger MJ. Sickness behavior in mice deficient in interleukin-6 during turpentine abscess and influenza pneumonitis. Am J Physiol. 1997; 272:R621R630. PMID: 9124487

62. Kaiser L, Fritz RS, Straus SE, Gubareva L, Hayden FG. Symptom pathogenesis during acute influenza: interleukin-6 and other cytokine responses. J Med Virol. 2001; 64: 262-268. PMID: 11424113

63. Arankalle VA, Lole KS, Arya RP, Tripathy AS, Ramdasi AY, Chadha MS et al. Role of host immune response and viral load in the differential outcome of pandemic H1N1 (2009) influenza virus infection in Indian patients. PLoS ONE. 2010; 5:e13099. doi: 10.1371/journal.pone.0013099 PMID: 20957032

64. Watanabe T, Watanabe S, Kawaoka Y. Cellular networks involved in the influenza virus life cycle. Cell Host Microbe. 2010; 7:427-39. doi: 10.1016/j.chom.2010.05.008 PMID: 20542247

65. Cornelissen JB, Post J, Peeters B, Vervelde L, Rebel JM. Differential innate responses of chickens and ducks to low-pathogenic avian influenza. Avian Pathol. 2012; 41(6):519-29. doi: 10.1080/03079457. 2012.732691 PMID: 23237364

66. Maughan MN, Dougherty LS, Preskenis LA, Ladman BS, Gelb J Jr, Spackman EV et al. Transcriptional analysis of the innate immune response of ducks to different species-of-origin low pathogenic $\mathrm{H} 7$ avian influenza viruses. Virol J. 2013; 10:94. doi: 10.1186/1743-422X-10-94 PMID: 23521892

67. Smith J, Smith N, Yu L, Paton IR, Gutowska MW, Forrest HL et al. A comparative analysis of host responses to avian influenza infection in ducks and chickens highlights a role for the interferon-induced transmembrane proteins in viral resistance.BMC Genomics. 2015; 16:574. doi: 10.1186/s12864-0151778-8 PMID: 26238195 